\title{
Erratum to: Pre-travel malaria chemoprophylaxis counselling in a public travel medicine clinic in São Paulo, Brazil
}

Tânia do Socorro Souza Chaves ${ }^{1,2,7^{*}}$, Wuelton Marcelo Monteiro ${ }^{4,5}$, Jessé Reis Alves ${ }^{2}$, Marcus Lacerda ${ }^{4,6}$ and Marta Heloisa Lopes ${ }^{3}$

\section{Erratum to: Malar J (2017) 16:64 \\ DOI 10.1186/s12936-017-1713-3}

After publication of the original article [1], it came to the authors' attention that Fig. 1 was annotated in Portuguese. The version of the Figure created using English is published in this erratum (Fig. 1).

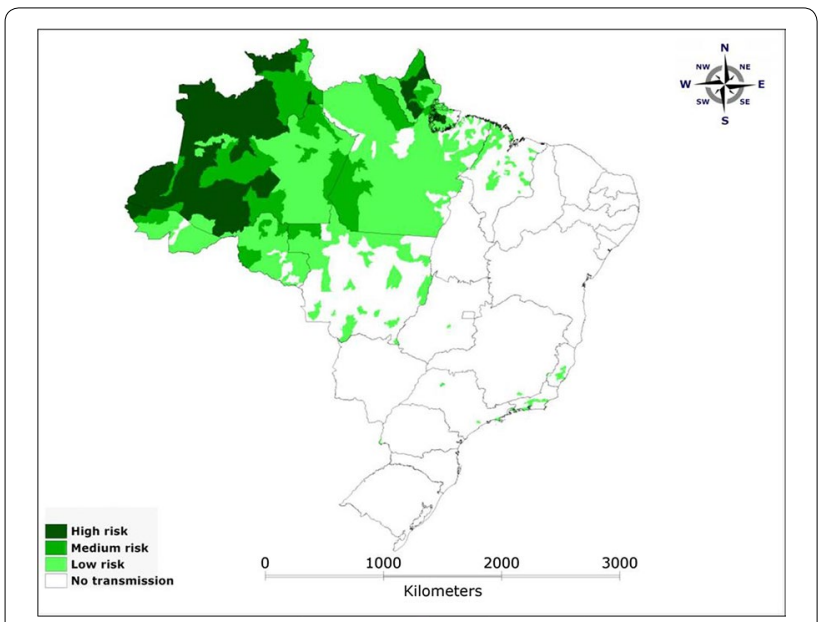

Fig. 1 Areas at risk of malaria transmission in Brazil [2]. Source: National Malaria Control Programme/Ministry of Health of Brazil

\begin{abstract}
Author details
${ }_{1}^{1}$ Parasitology Section, Evandro Chagas Institute, Rodovia BR-316, Km 7 s/n, Levilândia, Ananindeua, PA 67030-300, Brazil. ${ }^{2}$ Travel Medicine Division, Emilio Ribas Institute of Infectious Diseases, Av. Doutor Arnaldo 165, Cerqueira César, São Paulo 01246-900, Brazil. ${ }^{3}$ Departamento de Doenças Infecciosas, Faculdade de Medicina da Universidade de São Paulo, Av. Dr. Arnaldo 455, Cerqueira César, São Paulo, SP 01246-903, Brazil. ${ }^{4}$ Fundação de Medicina Tropical Dr. Heitor Vieira Dourado, Manaus, Brazil. ${ }^{5}$ Universidade do Estado do Amazonas, Manaus, Brazil. ${ }^{6}$ Instituto Leônidas \& Maria Deane (Fiocruz-Amazônia), Manaus, Brazil. ${ }^{7}$ Disciplina de Doenças Infecciosas e Parasitárias, Faculdade de Medicina, Universidade Federal do Pará, Av. Generalíssimo Deodoro, 01-Umarizal, Belém, PA 66050-160, Brazil.
\end{abstract}

The online version of the original article can be found under doi:10.1186/s12936-017-1713-3.

Published online: 17 March 2017

\section{References}

1. Chaves TSS, Monteiro WM, Alves JR, Lacerda M, Lopes MH. Pre-travel malaria chemoprophylaxis counselling in a public travel medicine clinic in São Paulo, Brazil. Malar J. 2017;16:64. doi:10.1186/s12936-017-1713-3.

2. http://portalarquivos.saude.gov.br/images/pdf/2017/fevereiro/32/Mapaderisco_malaria_2015.pdf. Accessed 6 Mar 2017.

\footnotetext{
*Correspondence: tania.chaves@uol.com.br

${ }^{1}$ Parasitology Section, Evandro Chagas Institute, Rodovia BR-316,

Km 7 s/n, Levilândia, Ananindeua, PA 67030-300, Brazil

Full list of author information is available at the end of the article
} provided you give appropriate credit to the original author(s) and the source, provide a link to the Creative Commons license, and indicate if changes were made. The Creative Commons Public Domain Dedication waiver (http://creativecommons.org/ publicdomain/zero/1.0/) applies to the data made available in this article, unless otherwise stated. 